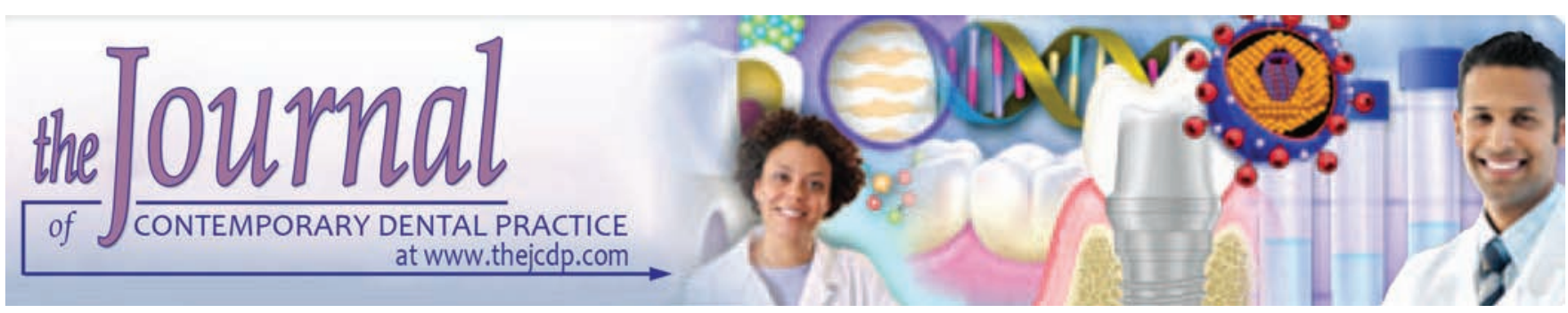

\title{
Comparison of Microleakage of Class V Cavities restored with the Embrace WetBond Class V Composite Resin and Conventional Opallis Composite Resin
}

\author{
${ }^{1}$ Maryam Tavangar, ${ }^{2}$ Zahra Zohri, ${ }^{3}$ Hosein Sheikhnezhad, ${ }^{4}$ Shahrzad Shahbeig
}

\begin{abstract}
Purpose: This study was undertaken to compare the microleakage of class $\mathrm{V}$ cavities restored with the newly introduced Embrace WetBond class $\mathrm{V}$ (EWC) composite resin and conventional Opallis composite resin.
\end{abstract}

Materials and methods: In this in vitro study, class $V$ cavities were prepared on 30 extracted bovine incisors, with the gingival floor and the coronal margin of the cavities $1 \mathrm{~mm}$ apical and coronal to the cementoenamel junction (CEJ) respectively. The cavities measured $3 \mathrm{~mm}$ in length, $2 \mathrm{~mm}$ in width, and $1.5 \mathrm{~mm}$ in depth. The teeth were randomly divided into two groups. In group I, the cavities were restored with Opallis composite resin in association with ExciTE adhesive system (total-etch); in group II, the EWC composite resin was used for restorations. After 500 thermocycling procedures, the teeth were immersed in $0.5 \%$ fuchsin solution for 24 hours. Then, the samples were placed within a polyester model and sectioned in the buccolingual direction. The samples were evaluated under a stereomicroscope at $\times 30$ for the penetration of dye. The enamel and dentin margins were evaluated separately. To test ordinal results, we used nonparametric statistical methods. To find out whether each independent composite groups I and II came from the same populations, we used Mann-Whitney $U$ test and to compare two related samples' coronal margin and gingival margin, Wilcoxon signed-rank test was used.

Results: There was significantly more microleakage in group II at both the enamel and dentin margins (coronal margin: $p=0.04$; gingival margin: $p=0.21$ ). In both groups, microleakage at

\footnotetext{
${ }^{1}$ Department of Operative Dentistry, Dental School, Guilan University of Medical Sciences, Rasht, Islamic Republic of Iran

${ }^{2,4}$ Department of Periodontics, School of Dentistry, Shahed University, Tehran, Islamic Republic of Iran

${ }^{3}$ Department of Prosthodontics, School of Dentistry, Guilan University, Rasht, Islamic Republic of Iran

Corresponding Author: Zahra Zohri, Department of Periodontics School of Dentistry, Shahed University, Tehran, Islamic Republic of Iran, Phone: +982188959210, e-mail: Z.Zohri@yahoo.com
}

gingival margins was significantly higher than that at coronal margins (group I: $p=0.008$; group II: $p=0.26$ ).

Conclusion: Despite the high speed and the short process of restoration with Embrace WetBond, it is not a reliable restorative material for class $\mathrm{V}$ cavities due to its inadequate marginal seal.

Keywords: Composite resin, Embrace WetBond, Marginal adaptation, Microleakage, Tooth bonding.

How to cite this article: Tavangar M, Zohri Z, Sheikhnezhad H, Shahbeig S. Comparison of Microleakage of Class V Cavities restored with the Embrace WetBond Class V Composite Resin and Conventional Opallis Composite Resin. J Contemp Dent Pract 2017;18(10):867-873.

Source of support: Nil

Conflict of interest: None

\section{INTRODUCTION}

Currently, use of composite resins is on the increase as a result of even increasing demands by patients for tooth-colored restorations. These restorations have some disadvantages despite their advantages, such as esthetic appearance, bonding to tooth structure, more conservative preparation of cavities that results in the preservation of tooth structure, and thermal insulation. The majority of problems in relation to composite resin restorations stem from polymerization shrinkage directly and indirectly. ${ }^{1,2}$ Such shrinkage results in enamel cracks, marginal gaps, and open margins, the important consequence of which is microleakage. ${ }^{3,4}$ Microleakage is the chief reason for tooth sensitivity and recurrent caries beneath the restoration, and, therefore, it is the main reason for the failure of treatment and replacement of composite resin restorations. ${ }^{1,5,6}$ Microleakage is attributed to various factors including the presence of gaps between the tooth structure and the restorative material, dentinal fluid, polymerization shrinkage, etc. ${ }^{7,8}$ Bacteria can proliferate and survive 
at marginal gaps, particularly noticeable on the walls that have a weaker bond, such as the dentinal margins compared with enamel margins, due to inadequate seal. In class $\mathrm{V}$ cavities, in particular, due to the difficulty of access, selection of a suitable restorative material is a controversial issue. ${ }^{5}$ Isolation is difficult at gingival margins, and a complete seal should be considered the most important factor for the success and durability of such restorations. ${ }^{7,9,10}$ A large number of studies have focused on composite resins and various bonding systems, which has resulted in significant advances in the type of the material used for tooth-colored restorations, the quality of the bond, and the techniques used for their clinical application. Recent advances in bonding systems have resulted in a decrease in procedural steps and simplification of these steps. For example, self-etch bonding systems or one-step bonding agents have prompted dentists to use these systems due to ease of the procedural steps and patient comfort. However, more attention should be paid to the quality of the bond than the ease of the procedural steps. In addition, subsequent to the introduction of bonding agents, many attempts were made to compare their properties to minimize microleakage, technique sensitivity, and the number of clinical steps, which resulted in the introduction of self-adhesive materials. Therefore, to simplify treatment, a new type of composite resin has been introduced recently, and the manufacturer claims that there is no need for acid etching and bonding on dentin surfaces with the use of this composite resin and it can itself form a chemical and micromechanical bond with tooth structures; however, etching of enamel surfaces has been recommended. ${ }^{7,11}$ This composite resin has been named EWC and is a hydrophilic resin that has exhibited excellent sealing ability, adaptation with tooth structure, and a strong bond; it has also exhibited less microleakage compared with conventional techniques of composite resin restorations. It is the first wet bond composite resin and has specifically been designed for class V cavities. ${ }^{12}$ The present study was designed to compare microleakage in class V cavities restored with EWC and Opallis composite resins to minimize microleakage in such restorations.

\section{MATERIALS AND METHODS}

Thirty permanent bovine incisors were included in the present in vitro study. The teeth were free of any caries or defects. The teeth were cleaned of any tissue remnants and stored in tap water during the study procedures.

\section{Cavity Preparation}

First, all the teeth were cleaned with a mixture of pumice powder and distilled water. The teeth were stored in a refrigerated saline solution for a maximum of 3 months as recommended by the International Organization for Standardization norms (ISO guidance on testing of adhesion to tooth structure; International Organization for Standardization. TR 11405, 1-4, Geneva, Switzerland, 1994). Then, class V cavities were prepared on the labial surfaces with a \#0.9 diamond bur (SS White, Lakewod, NJ, USA) in a high-speed handpiece under air and water spray by one operator. A new bur was used for every five cavities. The cavities measured $1.5 \mathrm{~mm}$ in depth, $3 \mathrm{~mm}$ in length, and $2 \mathrm{~mm}$ in width. The cavities were confined within the distal and mesial line angles of the teeth. The occlusal margin of the cavities was placed $1 \mathrm{~mm}$ coronal to the $\mathrm{CEJ}$, and the gingival margin was placed $1 \mathrm{~mm}$ apical to the CEJ (Fig. 1). Before cavity preparation procedures, the cavity outlines were drawn on each tooth with the use of a pencil. The teeth were randomly divided into two groups. In group I, Opallis composite resin (manufactured by FGM) and ExciTE bonding agent (manufactured by Vivadent) were used. In group II (the case group), EWC composite resin was used according to manufacturer's instructions.

\section{Restoration}

\section{Group I}

The enamel margins of the cavities were etched with 35\% phosphoric acid (Ultra Etch) for 15 seconds, followed by etching of the dentin surface for 10 seconds. After rinsing of the cavity for 10 seconds, they were blot-dried, which involved placing a cotton pellet within the cavity to cover the dentin surface and completely dry the enamel. Then, based on manufacturer's instructions, two layers of ExciTE bonding agent (a fifth-generation bonding agent manufactured by Vivadent) were applied to all the cavity surfaces. After 15 seconds, the first layer was

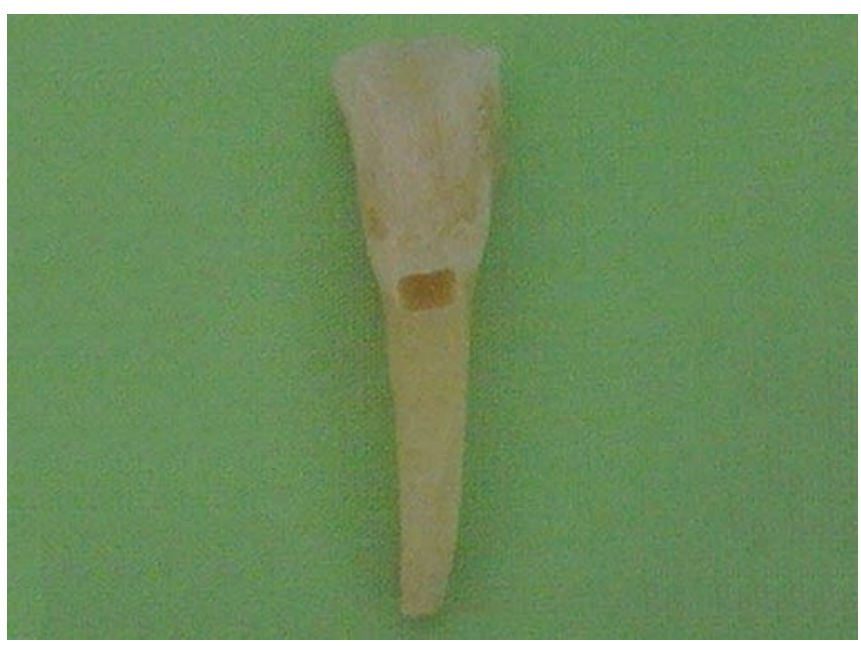

Fig. 1: Cavity preparation 
thinned with a gentle current of air from the air syringe. Then, the second layer was applied and thinned by the air syringe, followed by light-curing with Litex 680 (Dentamerica Inc, City of Industry, California), a lightcuring unit at a wavelength of $500 \mathrm{~nm}$. Subsequently, Opallis composite resin (Lot: 100212, A3 Dents Care Ltd, Brazil) was placed in the cavity using the incremental technique. The first layer, measuring $0.5 \mathrm{~mm}$ in thickness, was placed in the gingival half of the cavity and light-cured for 20 seconds. The subsequent layers were placed in $1 \mathrm{~mm}$ thicknesses and each layer was lightcured for 20 seconds. The last layer was light-cured for 40 seconds.

\section{Group /I}

A small groove was prepared with a diamond bur in a high-speed handpiece under air and water cooling at the incisal edge of all the tooth samples as an indicator. The enamel margins of the cavities were etched with 35\% phosphoric acid, rinsed, and dried. Care was exercised not to desiccate dentin. Therefore, the dentin surface was covered with a cotton pellet and was not subjected to the air syringe. Then, as described for group I, EWC composite resin (Pulpdent Corporation) was placed in the cavity using the incremental technique. The final layer was light-cured for 40 seconds. At the end of the restorative procedures in each group, all the samples were stored in tap water at room temperature for 24 hours, and then excess restorative materials were removed with finishing burs in a high-speed handpiece under water spray and the restorations were polished. All the procedures were carried out by one operator to eliminate the confounding factors as far as possible.

\section{Thermocycling}

Then, the samples were thermocycled in a special device manufactured under orders from the Faculty of Dentistry, Shahed University, Iran. The procedure consisted of 500 thermal cycles in water baths at $5 / 55^{\circ} \mathrm{C}$, with a dwell time of 30 seconds and a transfer time of 15 seconds. At the end of the procedures, the teeth were once again stored in tap water. The apices of the teeth were sealed with Fuji Plus self-cured glass-ionomer (manufactured by GS), and all the tooth surfaces were covered with two layers of nail varnish except for $1 \mathrm{~mm}$ around the restoration margins to confine dye penetration to the restoration margins. Subsequently, the teeth were stored in $0.5 \%$ fuchsin solution for 24 hours to evaluate microleakage. To prepare $0.5 \%$ concentration of fuchsin, $0.5 \mathrm{~g}$ of fuchsin powder was mixed with $99.5 \mathrm{~mL}$ of tap water. After 24 hours, the teeth were retrieved from the fuchsin solution and thoroughly rinsed under tap water to eliminate

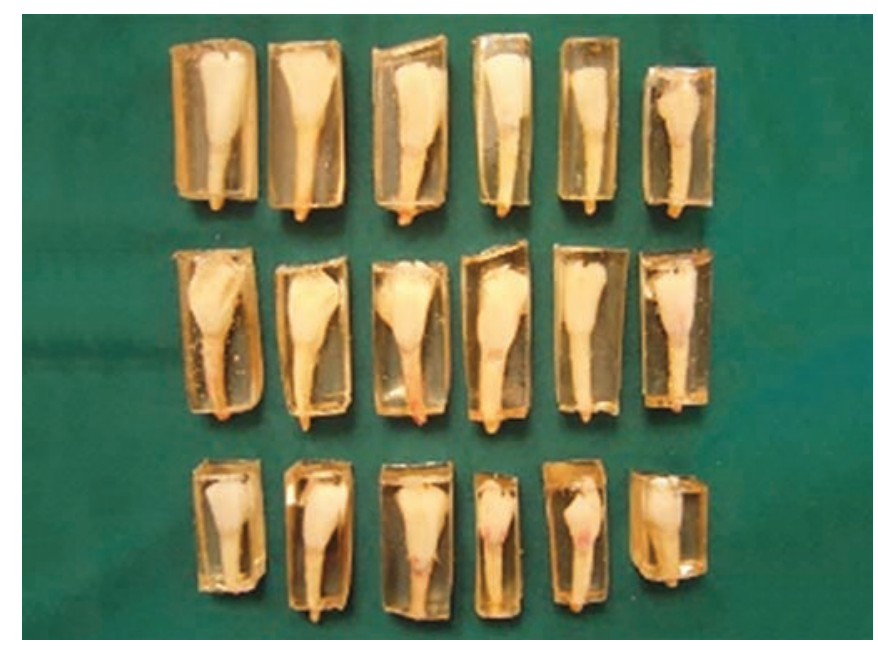

Fig. 2: Plastic models preparation

all the residual solution. Then, the nail varnish layers were removed from the tooth surfaces gently with the use of a spatula. Then, the teeth were placed within plastic models in preparation for sectioning.

\section{Preparation of the Plastic Models}

To prepare the plastic models, first, a hollow resin cube was prepared that was almost the size of the teeth. Then, the cube was filled with polyester material as follows (Maxi Rubber, São Paulo, SP, Brazil): First, a 4 mL syringe was used to transfer polyester resin and a drop of Brilliant cobalt into a metallic container and thoroughly mixed. Then, $0.2 \mathrm{~mL}$ of the shining Brilliant peroxide was added to the previous mixture and mixed thoroughly again (Fig. 2). It should be pointed out that accuracy and concentration during filling the wax patterns are very important to prevent bubble formation because after the setting process of the polyester material, bubbles result in the formation of cracks and fracture of the plastic model. The teeth should be placed within the mixture before the polyester material enters the gel phase. After setting which usually takes 20 to 30 minutes, the models should be retrieved from the models. The steps above were repeated for all the samples. All the samples in both groups were numbered.

\section{Sectioning}

For buccolingual section of each sample, a line was drawn with a graphic pen passing over the mesiodistal midline of the restoration. Each sample was split into two halves using a disk (Resista Omegna 68, Italy) under water coolant.

\section{Assessment}

The sections were prepared for the evaluation of dye penetration under a stereomicroscope at ×30 (SMZ 1500, 


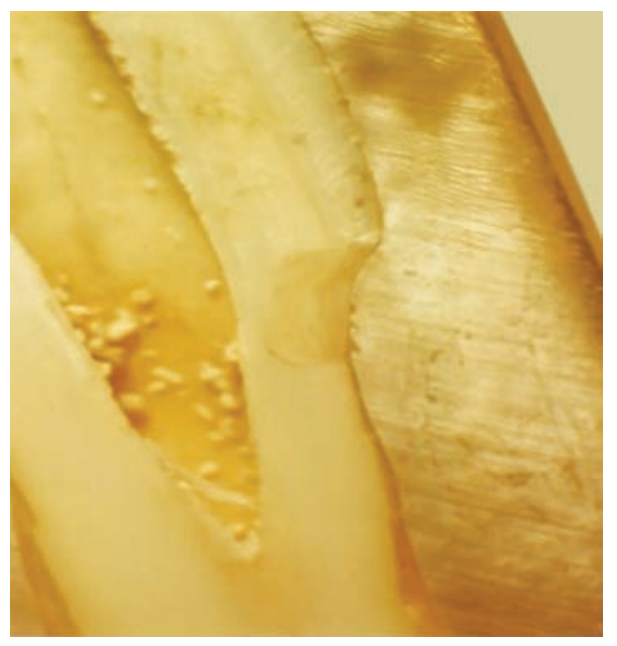

Fig. 3: Dye penetration in control group

Nikon, Kanagawa, Japan). Penetration of dye was evaluated at occlusal and gingival margins, and linear microleakage of the samples was scored as follows:

- Score 0: No dye penetration.

- Score 1: Dye penetration up to half of the depth or less than that on the occlusal and gingival walls.

- Score 2: Dye penetration up to more than half of the cavity without involving the axial wall.

- Score 3: Dye penetration involving the axial wall.

Such evaluation was carried out in both halves of each sample and twice for each half and the higher score was recorded (Figs 3 and 4).

\section{Statistical Analysis}

To test ordinal results, we used nonparametric statistical methods. To find out whether each independent composite group I and II came from the same population, we used Mann-Whitney U test, and to compare two related samples, coronal margin and gingival margin, Wilcoxon signed-rank test was used.

Table 1: Distribution of microleakage at coronal margins in groups I and II

\begin{tabular}{lllll}
\hline & & \multicolumn{2}{c}{ Groups } & \\
\cline { 3 - 4 } & & \multicolumn{1}{c}{ I } & Total \\
\hline Score 0 & $\mathrm{n}$ & 8 & 13 & 21 \\
& Percent & $53.3 \%$ & $86.7 \%$ & $70 \%$ \\
Score 1 & $\mathrm{n}$ & 3 & 1 & 4 \\
& Percent & $20 \%$ & $6.7 \%$ & $13.3 \%$ \\
Score 2 & $\mathrm{n}$ & 0 & 1 & 1 \\
& Percent & $0 \%$ & $6.7 \%$ & $3.3 \%$ \\
Score 3 & $\mathrm{n}$ & 4 & 0 & 4 \\
& Percent & $26.7 \%$ & $0 \%$ & $13.3 \%$ \\
Total & $\mathrm{n}$ & 15 & 15 & 30 \\
& Percent & $100 \%$ & $100 \%$ & $100 \%$ \\
\hline
\end{tabular}

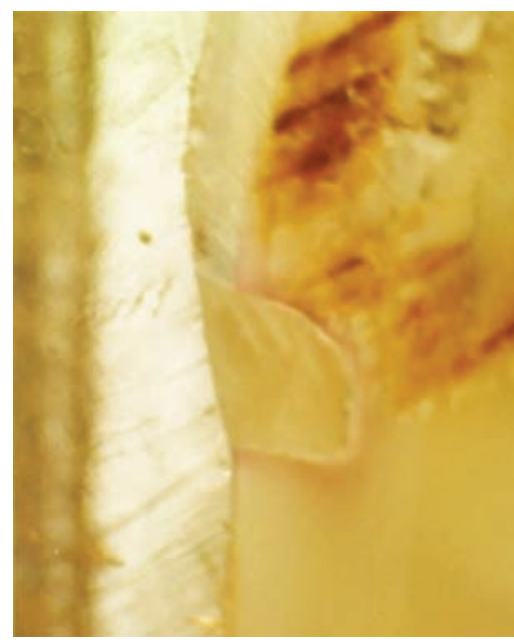

Fig. 4: Dye penetration in test group

\section{RESULTS}

Tables 1 and 2 present the mean microleakage scores of both gingival and coronal margins in each group. Table 3 presents the results of comparisons made between the two groups with Mann-Whitney U test. As shown by the results, there was significantly more microleakage at occlusal margins in group II (the case group; $p=0.04$ ). In addition, there was significantly more microleakage at gingival margins in group II ( $p=0.02)$. Wilcoxon's signed-rank test was used to compare microleakage between gingival and coronal margins in each group. In each group, microleakage at gingival margins was significantly more than that at coronal margins (Table 4).

\section{DISCUSSION}

One of the disadvantages of composite rein restorations is their long and complicated procedural steps. Composite resin restorations are time-consuming and comprise several steps with high technique sensitivity. Complete isolation, placement, and correct application of

Table 2: Distribution of microleakage at gingival margins in groups I and II

\begin{tabular}{lllll}
\hline & & \multicolumn{2}{c}{ Groups } & \\
\cline { 3 - 4 } & & $I$ & \multicolumn{1}{c}{ Total } \\
\hline Score 0 & $\mathrm{n}$ & 3 & 4 & 7 \\
& Percent & $20 \%$ & $26.7 \%$ & $23.3 \%$ \\
Score 1 & $\mathrm{n}$ & 3 & 9 & 12 \\
Score 2 & Percent & $20 \%$ & $60 \%$ & $40 \%$ \\
& $\mathrm{n}$ & 1 & 2 & 3 \\
Score 3 & $\mathrm{n}$ & $6.7 \%$ & $13.3 \%$ & $10 \%$ \\
& Percent & 8 & 0 & 8 \\
Total & $\mathrm{n}$ & $53.3 \%$ & $0 \%$ & $26.7 \%$ \\
& Percent & 15 & 15 & 30 \\
& & $100 \%$ & $100 \%$ & $100 \%$ \\
\hline
\end{tabular}


Comparison of Microleakage of Class V Cavities

Table 3: Comparison of microleakage in groups I and II

\begin{tabular}{lllllll}
\hline \multicolumn{2}{l}{ Group } & $N$ & Mean & $Z$ & $P$ & Result \\
\hline I & Coronal & 15 & 0.2 & -2.05 & 0.04 & Sig \\
II & margin & 15 & 2.2667 & & & \\
I & Gingival & 15 & 0.8667 & -2.312 & 0.021 & Sig \\
II & margin & 15 & 0.4667 & & & \\
\cline { 3 - 6 }
\end{tabular}

the materials and application of acid-etching, primer and the adhesive on the tooth structure require great skill and concentration. ${ }^{1}$ It should also be pointed out that composite resins exhibit at least $2 \%$ of polymerization shrinkage that might result in postoperative sensitivity, recurrent caries, and finally pulpal inflammation. ${ }^{13}$ Studies have predominantly shown that microleakage is significantly higher at the gingival margins of composite resin restorations that are apical to the CEJ. This is attributed to the fact that polymerization shrinkage is higher toward the center of the restorative material mass, toward the light source, and toward the bonded surface (composite resin-enamel interface).${ }^{13}$ Human teeth are the first choice for in vitro studies; however, bovine teeth have yielded microleakage results similar to those of human teeth and it is easy to collect them. ${ }^{14}$ Since, it is difficult to collect a large number of sound human teeth, in the present study, bovine teeth were used as an alternative. ${ }^{15}$ Different techniques have been used for the evaluation of microleakage in different studies. However, the most commonly used technique is dye penetration through the restorative material-tooth structure interface. Although it is an easy, inexpensive, and fast technique, it depends on the concentration and accuracy of the researcher for observing the results in the samples. ${ }^{14}$ Differences in procedural steps have made it difficult to compare the results reported by different researchers. Despite advances in the technologies used for the manufacturing of restorative materials and adhesive agents, microleakage takes place in the long term around restorations. ${ }^{15}$ Since the success rate has been low in relation to the elimination of or decrease in microleakage, attempts have been made to simplify the process of restoration with composite resins. ${ }^{16,17}$ Of all the composite resins available on the market, EWC is a new type of composite resin introduced as an alternative for time-consuming processes of composite resin restorations; however, extensive studies have not been carried out on it. The manufacturer claims that with the use of EWC, there is no need for acid etching and bonding on the dentin surface, and the composite resin itself can form a chemical and micromechanical bond with the tooth structure. A study evaluated microleakage of this material based on a request by the manufacturer and reported that this technique exhibited less microleakage compared with conventional composite resin
Table 4: Comparison of microleakage between gingival and coronal margins

\begin{tabular}{llllll}
\hline \multicolumn{1}{l}{ Group } & Mean & $Z$ & $P$ & Result \\
\hline I & Coronal margin & 0.2 & -2.673 & 0.008 & Sig \\
& Gingival margin & 0.86 & & & \\
II $\quad$ Coronal margin & 1.26 & -2.226 & 0.026 & Sig \\
& Gingival margin & 2.46 & & & \\
\hline
\end{tabular}

restorations; ${ }^{11}$ however, the results of the present study did not confirm this. Concomitant with such an advance, self-adhesive cements have been marketed too and have attracted a lot of attention. These resin cements do not require bonding and preparation of the tooth surface. The results of studies by Ibarra have shown that one of these resin cements, namely, Unicem, has low ability to etch the smear layer covering the enamel and, therefore, results in a decrease in the micromechanical retention of the cement. A decrease in the micromechanical retention on enamel might be due to the high viscosity of the cement after mixing it or due to the insufficient time available for the cement, before curing, to react with the tooth structure. In such a case, neutralizing reactions occur rapidly, and the initial $\mathrm{pH}(\mathrm{pH}<2)$ will not be suitable for etching the enamel surface. Evaluation of the samples under an electron microscope has demonstrated a decrease in the thickness of the hybrid layer, which might be a piece of evidence indicating a decrease in bond strength. ${ }^{18,19}$ Grandini et $\mathrm{al}^{20}$ carried out a study on cemented fiberreinforced composite posts with five different types of adhesive cements, concluding that despite the ease and high rate of the procedural steps with experimental GC and Unicem cements, the short setting time and the relatively high viscosity of these cements might compromise marginal adaptation along the root canal and even result in formation of bubbles, consistent with the results of the present study. ${ }^{21}$ To explain this further, since no adhesive system is used with EWC composite resin and also due to its high viscosity, proper wetting does not occur on the tooth surface and marginal adaptation decreases. In general, in the study carried out by Francesca, selfadhesive cements evaluated were not recommended due to their poor marginal seal and low bond strength compared with other cements. ${ }^{22}$ Radovic et al ${ }^{23}$ evaluated the efficacy of self-adhesive cements and reported that the adhesive capacity of self-adhesive cements to dentin was comparable with that of other resin cements; however, these cements exhibited a weak bond to enamel. These results were confirmed by Ibarra et al, ${ }^{19}$ De Munck et $\mathrm{al}_{1}{ }^{22}$ and Schenke et $\mathrm{al}^{24}{ }^{24}$ Studies carried out based on requests by the manufacturer of Unicem cement (3M) in 2002 compared Unicem with composite resin cements (Variolink II) and compomer (Dyract) and resin cements (Panavia F) in relation to marginal seal at enamel and 
dentin, with the minimum microleakage being recorded with Panavia F cement and maximum with modified GI and compomer. Unicem cement exhibited microleakage results comparable with those of Panavia. ${ }^{25,26}$ Shafiei et $\mathrm{al}^{6}$ reported microleakage of self-adhesive class $\mathrm{V}$ composite resins in deciduous and permanent teeth with the use of self-adhesive EWC composite resin. ${ }^{13}$ Sadeghi ${ }^{9}$ compared microleakage of self-adhesive flowable composite resin with other flowable resin materials and reported better occlusal marginal seal with EWC composite reins in association with bonding compared with no bonding; however, no significant differences were detected at cervical margins. Therefore, despite the efforts to decrease the number of procedural steps and simplify the restorative process, based on the results of different studies, it still appears that the etch-and-rinse technique is the most effective technique to achieve a durable bond to tooth structure. ${ }^{27-29}$ Therefore, shortening the restorative process, irrespective of microleakage, cannot be considered a significant breakthrough, and this indicates the importance of dentin-bonding systems in the marginal seal and decease in microleakage. It should be pointed out that since the present study was carried out in vitro, it is recommended that long-term in vivo studies be carried out under similar conditions. Since there were problems with the technique used in the present study to fabricate the plastic models, researchers intending to carry out such studies are recommended to fabricate transparent plastic models with the use of orthodontic acrylic resins because in addition to the ease of fabrication, they are better than polyester and can be sectioned by a machine.

\section{CONCLUSION}

Despite the high rate of the procedure and its short time when EWC composite resin is used, it cannot be a reliable restorative material for class $\mathrm{V}$ cavities due to inadequate marginal seal.

\section{REFERENCES}

1. Parameswaran A. Sturdevant's art and science of operative dentistry. J Conserv Dent 2013 Sep;16(5):480.

2. Santos AJ, Lisso MT, Aguiar FH, França FM, Lovadino JR. Effect of stepped exposure on quantitative in vitro marginal microleakage. J Esthet Restor Dent 2005 Jul;17(4):236-242, discussion 243.

3. Bektas OO, Eren D, Akin EG, Akin H. Evaluation of a self-adhering flowable composite in terms of micro-shear bond strength and microleakage. Acta Odontol Scand 2013 May-Jul;71(3-4):541-546.

4. Tavangar M, Tayefeh Davalloo R, Darabi F, Karambin M, Kazemi R. A comparative evaluation of microleakage of two low-shrinkage composites with a conventional resin composite: an in vitro assessment. J Dent (Shiraz) 2016 Mar;17(1):55-61.
5. Kasraei S, Azarsina M, Majidi S. In vitro comparison of microleakage of posterior resin composites with and without liner using two-step etch-and-rinse and self-etch dentin adhesive systems. Oper Dent 2011 Mar-Apr;36(2):213-221.

6. Shafiei L, Mojiri P, Ghahraman Y, Rakhshan V. Microleakage of a self-adhesive class $\mathrm{V}$ composite on primary and permanent dentitions. J Contemp Dent Pract 2013 May;14(3):461-467.

7. Geerts S, Bolette A, Seidel L, Guéders A. An in vitro evaluation of leakage of two etch and rinse and two self-etch adhesives after thermocycling. Int J Dent 2012 Mar;2012:852841.

8. Vinay S, Shivanna V. Comparative evaluation of microleakage of fifth, sixth, and seventh generation dentin bonding agents: an in vitro study. J Conserv Dent 2010 Oct;13(3):136-140.

9. Sadeghi M. An in vitro microleakage study of class V cavities restored with a new self-adhesive flowable composite resin versus different flowable materials. Dent Res J (Isfahan) 2012 Jul-Aug;9(4):460-465.

10. Sánchez-Ayala A, Farias-Neto A, Vilanova LS, Gomes JC, Gomes OM. Marginal microleakage of class V resin-based composite restorations bonded with six one-step self-etch systems. Braz Oral Res 2013 May-Jun;27(3):225-230.

11. Hoffman ID. Advanced resin technology: embrace Wetbond. Spectrum 2005;4(1):68-76.

12. Singla R, Bogra P, Singal B. Comparative evaluation of traditional and self-priming hydrophilic resin. J Conserv Dent 2012 Jul-Sep;15(3):233-236.

13. El-Mowafy O, El-Badrawy W, Eltanty A, Abbasi K, Habib N. Gingival microleakage of class II resin composite restorations with fiber inserts. Oper Dent 2007 Jun;32(3):298-305.

14. Silveira de Araújo C, Incerti da Silva T, Ogliari FA, Meireles SS, Piva E, Demarco FF. Microleakage of seven adhesive systems in enamel and dentin. J Contemp Dent Pract 2006 Nov; 7(5):26-33.

15. Borges MA, Matos IC, Dias KR. Influence of two selfetching primer systems on enamel adhesion. Braz Dent J 2007;18(2):113-118.

16. Ernst CP, Cortain G, Spohn M, Rippin G, Willershausen B. Marginal integrity of different resin-based composites for posterior teeth: an in vitro dye-penetration study on eight resin-composite and compomer/adhesive combinations with a particular look at the additional use of flow-composites. Dent Mater 2002 Jun;18(4):351-358.

17. Guéders AM, Charpentier JF, Albert AI, Geerts SO. Microleakage after thermocycling of 4 etch and rinse and 3 self-etch adhesives with and without a flowable composite lining. Oper Dent 2006 Jul-Aug;31(4):450-455.

18. Cenci M, Demarco F, de Carvalho R. Class II composite resin restorations with two polymerization techniques: Relationship between microtensile bond strength and marginal leakage. J Dent 2005 Aug;33(7):603-610.

19. Ibarra G, Johnson GH, Geurtsen W, Vargas MA. Microleakage of porcelain veneer restorations bonded to enamel and dentin with a new self-adhesive resin-based dental cement. Dent Mater 2007 Feb;23(2):218-225.

20. Grandini S, Goracci C, Monticelli F, Tay FR, Ferrari M. Fatigue resistance and structural characteristics of fiber posts: threepoint bending test and SEM evaluation. Dent Mater 2005 Feb;21(2):75-82.

21. Zicari F, Couthino E, De Munck J, Poitevin A, Scotti R, Naert I, Van Meerbeek B. Bonding effectiveness and sealing ability of fiber-post bonding. Dent Mater 2008 Jul;24(7):967-977. 
22. De Munck J, Vargas M, Van Landuyt K, Hikita K, Lambrechts P, Van Meerbeek B. Bonding of an auto-adhesive luting material to enamel and dentin. Dent Mater 2004 Dec;20(10):963-971.

23. Radovic I, Monticelli F, Goracci C, Vulicevic ZR, Ferrari M. Self-adhesive resin cements: a literature review. J Adhes Dent 2008 Aug;10(4):251-258

24. Schenke F, Hiller KA, Schmalz G, Federlin M. Marginal integrity of partial ceramic crowns within dentin with different luting techniques and materials. Oper Dent 2008 Sep-Oct;33(5):516-525.

25. Behr M, Rosentritt M, Lang R, Regnet T, Handel G. Marginal adaptation of all-ceramic crowns using different lutingcements. J Dent Res 2002;81:A421-A421.
26. Rosentritt M, Behr M, Lang R, Handel G. Marginal adaptation of ceramic in lays using different types of cements. J Dent Res 2002;81:A36.

27. Owens BM, Johnson WW. Effect of insertion technique and adhesive system on microleakage of class $V$ resin composite restorations. J Adhes Dent 2005 Winter;7(4):303-308.

28. Pamir T, Türkün M. Factors affecting microleakage of a packable resin composite: an in vitro study. Oper Dent 2005 May-Jun;30(3):338-345.

29. Poskus LT, Placido E, Cardoso PE. Influence of adhesive system and placement technique on microleakage of resinbased composite restorations. J Adhes Dent 2004 Autumn;6(3): 227-232. 